\title{
High effort, low reward, and cardiovascular risk factors in employed Swedish men and women: baseline results from the WOLF Study
}

Richard Peter, Lars Alfredsson, Niklas Hammar, Johannes Siegrist, Töres Theorell, Peter Westerholm

\begin{abstract}
Study objective-To examine associations between measures of work stress (that is, the combination of high effort and low reward) and cardiovascular risk factors. Design-Cross sectional first screening of a prospective cohort study.

Setting and participants-The study was conducted among 5720 healthy employed men and women living in the greater Stockholm area aged 19-70 years. All analyses were restricted to subjects with complete data $(n=4958)$. The investigation of associations between indicators of effort-reward imbalance and cardiovascular risk factors was restricted to the age group 30-55 years $(n=3427)$.
\end{abstract}

Main results-Subjects reporting high effort and low reward at work had a higher prevalence of well known risk factors for coronary heart disease. After adjustment for relevant confounders, associations between a measure of extrinsic effort and reward (the effort-reward ratio) and hypertension (multivariate prevalence odds ratio (POR) 1.62-1.68), increased total cholesterol (upper tertile $220 \mathrm{mg} /$ d1) $(P O R=1.24)$ and the total cholesterol/ high density lipoprotein(HDL)cholesterol ratio (upper tertile 4.61)(POR 1.26-1.30) were found among men. Among women a measure of high intrinsic effort (immersion) was related to increased low density lipoprotein(LDL)-cholesterol (upper tertile $130 \mathrm{mg} / \mathrm{dl}$ )(POR 1.37-1.39). Analyses of variance showed increasing mean values of LDL cholesterol with an increasing degree of the effort-reward ratio among men and increased LDLcholesterol among women with high levels of intrinsic effort (upper tertile of immersion).

Conclusions-Findings lend support to the hypothesis that effort-reward imbalance represents a specific constellation of stressful experience at work related to cardiovascular risk. The relation was not explained by relevant confounders (for example, lack of physical exercise, body weight, cigarette smoking).

(F Epidemiol Community Health 1998;52:540-547)

Correspondence to:

Medical Sociology,

Heinrich-Heine University,

PO Box 1010 07, D-4000

Düsseldorf, Germany.

Accepted for publication 9 February 1998 achieved in measuring chronic stress at work and in demonstrating its adverse effects on cardiovascular health. ${ }^{1-5}$ Fewer studies have been carried out among women than among men. This became particularly clear when chronic work stress was assessed in terms of the job strain model developed and tested by Karasek, ${ }^{6}$ Karasek and Theorell, ${ }^{7}$ Johnson et $a l,{ }^{89}$ and others. ${ }^{10}$ This model claims that the experience of stress at work is confined to a specific job task constellation defined by high demands (such as work pressure) in combination with low control (such as low decision latitude, or holding a monotonous job). Chronic work stress among women was often attributed to the specific constellation of double exposure (work and home), to less continuous occupational careers or to different impact of work on the life course of women. ${ }^{11-13}$ This latter aspect is of special importance as the work role offers unique opportunities for social identity and self esteem in adult life, and, perhaps, more often so in men than in women.

To explore the health promoting and health adverse effects of self regulation in occupational life the model of effort-reward imbalance at work was developed and tested by Siegrist and his group. ${ }^{5}$ In summary, this model defines chronically stressful experience at work in terms of a mismatch between high effort spent and low reward received in occupational life. Two sources of effort are distinguished, an extrinsic source, the demands on the job, and an intrinsic source, the motivations of the individual worker in a demanding situation. In this latter regard, the concept of "need for control" as a personal pattern of coping with the demands at work was introduced (see Methods section). These efforts are spent as part of a socially organised exchange process where rewards are distributed by three transmitter systems: money, esteem, and job security or promotion prospects (so called occupational status control). Lack of reciprocity between costs and gains define a state of emotional distress with special propensity to autonomic arousal and cardiovascular risk. ${ }^{14}{ }^{15}$

Therefore it is of special interest to examine the effects of effort-reward imbalance on cardiovascular risk in men and women. More specifically, we ask whether effects of similar strength and consistency are observed in both sexes and whether they are attributed to the same type of high cost-low gain experience. In this latter regard the model defines two measures of a mismatch; firstly, the ratio between extrinsic efforts and rewards, and secondly, the sum score of "need for control" indicating individual work related 
commitments (see Methods section). Given the relative importance of work and career development (occupational rewards) in male middle aged populations it may well be that the first measure of mismatch is more important in explaining stress related health outcomes in men compared with women. Conversely, women spending high levels of intrinsic effort may be particularly vulnerable to stress related health outcomes.

This study reports baseline findings on associations between high effort, low reward, and cardiovascular risk obtained from the so called WOLF (Work, Lipids, Fibrinogen) Study. This study was started to analyse the role of adverse occupational conditions in cardiovascular risk and disease development in a large sample of employed Swedish men and women.

\section{Methods}

STUDY SAMPLE

Occupational health service units serving a population that was considered suitable for the aims of the study were invited to take part in the WOLF study as a cooperative centre. A total of 20 units accepted the invitation and three did not. The study population consisted of employees at almost 40 companies served by these 20 occupational health service units representing a number of different branches and a wide range of occupations. Subjects who were on a more or less permanent leave from the work place, for example, stationed abroad or chronically ill, were not included in the study population.

The data collection of the Stockholm part of the WOLF study started in November 1992 and ended in June 1995. At baseline screening 3250 men and 2470 women aged 19-70 years were included. This analysis is restricted to subjects aged 30 to 55 years. This was done because the strongest associations between indicators of chronic work stress and cardiovascular risk factors can be expected in this age group. $^{59}$

The group with complete data $(n=3427)$ includes $84.6 \%$ of the total sample in this age group $(n=4052)$. It was found that the prevalence of several cardiovascular risk factors and the prevalence of exposure (for example, indicators of high effort and low reward) were lower in the reduced sample as compared with the group which was excluded from statistical analyses because of unsystematic missing data. This selection bias might have caused an underestimation of the relation between indicators of effort-reward imbalance and cardiovascular risk factors (see Discussion section).

PSYCHOSOCIAL AND BIOBEHAVIOURAL

QUESTIONNAIRE DATA

All information on psychosocial and behavioural variables was assessed by an extensive questionnaire administered by the occupational health unit to each study subject for completion at home.

Effort-reward imbalance at work

The model of effort-reward imbalance was measured by Likert scaled and categorial items: intrinsic effort was assessed by a well tested questionnaire measuring "need for control", a critical personal style of coping with work demands. ${ }^{16}$ The questionnaire contains 29 dichotomous items describing excessive job involvement, positive and negative feelings and attitudes related to work commitment as measured by four unidimensional scales: (a) need for approval; (b) competitiveness and latent hostility; (c) impatience and disproportionate irritability; and (d) inability to withdraw from work obligations. These four scales were repeatedly found to load on a latent factor termed "immersion". Scores in the upper tertile of the latent factor immersion indicate a critically increased coping style, which was shown to predict premature manifestation of cardiovascular events. ${ }^{17}$

Measures of reward in this study were not fully identical with original wording of questions. However, given the close conceptual and semantical resemblence respective questions were used to construct proxy measures of low reward (see appendix 1). It is important to mention that these measures were defined before data analysis started. Questions measuring extrinsic effort (demands) concerned the frequency of being distressed by time pressure, responsibility, overtime work, and increasing responsibility during the past 12 months. Measures of occupational rewards covered the dimensions of esteem and reciprocal support, and of occupational status control (job insecurity, undesirable job change).

As stated in the Introduction section two measures of a mismatch between high effort and low reward were developed to identify the specific stressfulness of occupational experience as defined by this model. Firstly, we computed a ratio between the scales measuring extrinsic effort and reward respectively (see appendix 1) according to the following formula based on dichotomised items: e / $(\mathrm{r} \times \mathrm{c})$

$\mathrm{e}=$ extrinsic effort sum score, counting the number of items indicating high extrinsic effort; $r=$ reversed reward sum score, counting the number of items indicating low reward; $\mathrm{c}=$ correction factor for different maximum numbers of items in the nominator and the denominator calculated as the total number of items in the nominator divided by the total number of items in the denominator.

As a result, a value of 1.0 indicates a balance between effort and reward. Values $<1.0$ indicate that rewards are higher than efforts, whereas values $>1.0$ indicate the critical high cost/ low gain condition.

Secondly, a sum score of the latent factor "immersion" was calculated, measuring the extent of intrinsic effort. We hypothesise that people scoring high on "immersion" usually spend an inadequately high amount of effort that is not met by externally defined rewards. This may be because of the fact that high need for control often induces an underestimation of demands and an overestimation of own coping resources. ${ }^{516}$ 
Table 1 Biobehavioural cardiovascular risk factors and psychosocial variables among a sample of employed Swedish men and women aged 19-70 years. (Mean and SD or frequency, $t$ value or $\chi^{2}$ )

\begin{tabular}{|c|c|c|c|}
\hline Variable & $\begin{array}{l}\text { Men } \\
(n=2656)\end{array}$ & $\begin{array}{l}\text { Women } \\
(n=2088)\end{array}$ & $t$ value or $\chi^{2}$ \\
\hline Age (y) & $41.5 \quad(11.0)$ & $41.2(10.7)$ & 0.90 \\
\hline Systolic blood pressure (mm Hg) & $123.1 \quad(14.2$ & $118.2(14.9)$ & $11.38^{\star \star \star}$ \\
\hline Diastolic blood pressure $(\mathrm{mm} \mathrm{Hg})$ & $74.7 \quad(9.8)$ & $71.7 \quad(9.8)$ & $10.74^{\star \star \star}$ \\
\hline Total cholesterol (mg/dl) & $210.4 \quad(41.9)$ & $202.1 \quad(38.6)$ & $7.04^{\star \star \star}$ \\
\hline LDL-cholesterol (mg/dl) & $135.2(38.4)$ & $119.9 \quad(35.6)$ & $14.16^{\star \star \star}$ \\
\hline HDL-cholesterol (mg/dl) & $52.3(13.2)$ & $66.1(16.2)$ & $31.28^{\star \star \star}$ \\
\hline Total / HDL-cholesterol ratio & $4.2 \quad(1.3)$ & $3.2(1.0)$ & $30.66^{\star \star \star}$ \\
\hline Fibrinogen $(\mathrm{mg} / \mathrm{dl})$ & $(72.7)$ & $280.0 \quad(73.9)$ & $5.27^{\star}$ \\
\hline Body mass index $(\mathrm{BMI})\left(\mathrm{kg} / \mathrm{m}^{2}\right)$ & 25.2 & 23.8 & $12.99^{\star \star \star}$ \\
\hline Cigarette smoking (numbers/day) & $3.1(7.2)$ & $3.7(7.0)$ & $2.49^{\star \star}$ \\
\hline Lack of physical exercise $(<2 /$ week $)$ & $60.5 \%$ & $57.1 \%$ & $15.32^{\star \star \star}$ \\
\hline Socioeconomic group (white collar) & $59.2 \%$ & $85.3 \%$ & $272.49^{\star \star \star}$ \\
\hline \multicolumn{4}{|l|}{ Extrinsic effort and reward: } \\
\hline Effort-reward ratio (range $0.01-2.0$ ) & $0.93(0.24)$ & $0.88(0.27)$ & $6.50^{\star \star \star}$ \\
\hline Effort-reward ratio $(>1)$ & $31.0 \%$ & $27.0 \%$ & $8.73^{\star \star}$ \\
\hline \multicolumn{4}{|l|}{ Intrinsic effort: } \\
\hline Immersion (range 0-29) & $10.6(4.4)$ & $10.9(4.5)$ & $2.51^{\star \star}$ \\
\hline Immersion (upper tertile) & $31.5 \%$ & $34.2 \%$ & $5.92^{\star}$ \\
\hline
\end{tabular}

${ }^{\star} \mathrm{p} \leqslant 0.05,{ }^{\star \star} \mathrm{p} \leqslant 0.01,{ }^{\star \star \star} \mathrm{p} \leqslant 0.001$.

Table 2 Frequency of the effort-reward ratio and immersion by sociodemographic information among a sample of employed Swedish men and women aged 30-55 years (frequency, $\chi^{2}$ )

\begin{tabular}{|c|c|c|}
\hline Variable & $\begin{array}{l}\text { Effort-reward ratio }(>1) \\
(n=655)\end{array}$ & $\begin{array}{l}\text { Immersion (upper tertile) } \\
(n=607)\end{array}$ \\
\hline \multicolumn{3}{|l|}{ Age } \\
\hline $30-40$ y $(n=804)$ & 34.6 & 32.7 \\
\hline $41-45$ y $(n=344)$ & 32.6 & 34.9 \\
\hline $46-50$ y $(n=417)$ & 35.0 & 28.8 \\
\hline \multirow{2}{*}{$51-55$ y $(n=348)$} & 34.2 & 29.9 \\
\hline & $\chi^{2}=0.58$ & $\chi^{2}=4.16$ \\
\hline \multicolumn{3}{|l|}{ Education/training } \\
\hline$<9$ y $(n=269)$ & 27.1 & 24.9 \\
\hline $10 \mathrm{y}(\mathrm{n}=368)$ & 29.6 & 24.7 \\
\hline $12 \mathrm{y}(\mathrm{n}=580)$ & 35.5 & 34.7 \\
\hline \multirow[t]{2}{*}{ university degree $(n=694)$} & 38.3 & 35.7 \\
\hline & $\chi^{2}=15.09^{\star \star}$ & $\chi^{2}=21.53^{\star \star \star}$ \\
\hline \multirow{4}{*}{$\begin{array}{l}\text { Socioeconomic group } \\
\text { blue collar }(n=675) \\
\text { white collar }(n=1238)\end{array}$} & & \\
\hline & 28.6 & 23.0 \\
\hline & 37.3 & 36.5 \\
\hline & $\chi^{2}=14.77^{\star \star \star}$ & $\chi^{2}=37.01^{\star \star \star}$ \\
\hline \multicolumn{3}{|l|}{ Women $(n=1514)$} \\
\hline Variable & $\begin{array}{l}\text { Effort-reward ratio }(>1) \\
(n=430)\end{array}$ & $\begin{array}{l}\text { Immersion (upper tertile) } \\
(n=512)\end{array}$ \\
\hline \multicolumn{3}{|l|}{ Age } \\
\hline $30-40$ y $(n=533)$ & 28.9 & 36.0 \\
\hline $41-45$ y $(n=334)$ & 21.6 & 33.8 \\
\hline $46-50$ y $(n=398)$ & 32.7 & 30.9 \\
\hline \multirow[t]{2}{*}{$51-55$ y $(n=249)$} & 32.9 & 33.7 \\
\hline & $\chi^{2}=13.47^{\star \star}$ & $\chi^{2}=2.67$ \\
\hline \multicolumn{3}{|l|}{ Education } \\
\hline$<9$ y $(n=190)$ & 22.8 & 27.8 \\
\hline $10 \mathrm{y}(\mathrm{n}=295)$ & 23.0 & 33.1 \\
\hline 12 y $(n=397)$ & 23.3 & 34.5 \\
\hline \multirow[t]{2}{*}{ university degree $(n=692)$} & 36.3 & 35.4 \\
\hline & $\chi^{2}=31.72^{\star \star \star}$ & $\chi^{2}=3.86$ \\
\hline \multicolumn{3}{|l|}{ Socioeconomic group } \\
\hline blue collar $(n=223)$ & 23.3 & 25.6 \\
\hline \multirow[t]{2}{*}{ white collar $(n=1291)$} & 29.9 & 35.2 \\
\hline & $\chi^{2}=4.00^{\star}$ & $\chi^{2}=7.97^{\star \star}$ \\
\hline
\end{tabular}

${ }^{\star} \mathrm{p} \leqslant 0.05,{ }^{\star \star} \mathrm{p} \leqslant 0.01,{ }^{\star \star \star} \mathrm{p} \leqslant 0.001$

\section{Biobehavioural data}

Information on health related behaviour (cigarette smoking, physical exercise, medication), diesase history and heredity factors concerning hypertension, hyperlipidaemia and coronary heart disease was obtained from well tested questions.

CLINICAL EXAMINATION DATA

A health examination was carried out at the occupational health service unit. This exami-
KEY POINTS

- High efforts in combination with low rewards define a critical constellation of work related stressful experience.

- In a healthy employed population effortreward imbalance is associated with cardiovascular risk factors (hypertension, atherogenic lipids).

- Effects in both sexes are of comparable strength, but because of different components of the effort-reward imbalance model.

nation involved measurements of height, weight, waist, hip, and blood pressure. Blood samples were collected for determination of blood lipids and fibrinogen. All subjects were asked to fast nine hours before the clinical examination.

Blood pressure was measured on the right arm in the supine position after five minutes rest. Measurements were made twice with a one minute intermission. Atherogenic lipids were measured enzymatically, high density lipoprotein (HDL)-cholesterol after precipitation with phospotungstic acid and magnesium chloride. Fibrinogen in plasma was determined by a spectrophotometric test. All analyses of blood lipids and fibrinogen were carried out at the same laboratory (CALAB Medical Laboratories AB, Stockholm, Sweden). The laboratory is accredited by SWEDAC (Swedish Board for Accreditation and Conforming Assessment).

In this study data analysis is based on categorial and continuous biomedical factors. Whereas the categorisation of blood pressure according to the WHO criteria is well established (systolic blood pressure > 160 and/or diastolic blood pressure $>95 \mathrm{~mm} \mathrm{Hg}$ ) we are aware that the international standardisation of cut points for abnormal atherogenic lipid and fibrinogen values is still under discussion. Therefore we based our decision of cut points with regard to these biomedical measures on recent epidemiological evidence indicating thresholds of clearly increased incidence of cardiovascular disease and on the distribution of the data. The thresholds based on epidemiological evidence are the upper tertile with respect to low density lipoprotein (LDL)cholesterol and total cholesterol (for example, in men LDL-cholesterol > $160 \mathrm{mg} / \mathrm{dl}$, total cholesterol > $220 \mathrm{mg} / \mathrm{dl}$ in both sexes). ${ }^{18}{ }^{19}$ For women the upper tertile of LDL-cholesterol started at $130 \mathrm{mg} / \mathrm{dl}$. With regard to fibrinogen empirical evidence again indicates a cut point at the upper tertile for both sexes, ${ }^{20-23}$ which corresponds to $290 \mathrm{mg} / \mathrm{dl}$ for men and 300 $\mathrm{mg} / \mathrm{dl}$ for women in our study. Concerning the total cholesterol/HDL-cholesterol ratio the upper tertile was chosen as a cut off point relating to the distribution of the data: 4.61 for men and 3.4 for women.

SOCIOECONOMIC DATA

Socioeconomic group was defined on the basis of information on occupation and education in 
Table 3 Frequency of the effort-reward ratio and immersion by cardiovascular risk factors in a sample of employed Swedish men and women aged 30-55 years $\left(\chi^{2}\right)$

\begin{tabular}{|c|c|c|c|c|c|c|c|c|c|c|}
\hline \multirow{2}{*}{$\begin{array}{l}\text { Men } \\
\text { Psychosocial variables }\end{array}$} & \multicolumn{10}{|c|}{ Cardiovascular risk factors } \\
\hline & \multicolumn{2}{|c|}{$\begin{array}{l}\text { Hypertension }(\geqslant 160 / 95 \\
\text { mm Hg) }\end{array}$} & \multicolumn{2}{|c|}{$\begin{array}{l}\text { Total cholesterol (upper } \\
\text { tertile } \geqslant 220 \mathrm{mg} / \mathrm{dl} \text { ) }\end{array}$} & \multicolumn{2}{|c|}{$\begin{array}{l}\text { LDL-cholesterol (upper } \\
\text { tertile } \geqslant 160 \mathrm{mg} / \mathrm{dl} \text { ) }\end{array}$} & \multicolumn{2}{|c|}{$\begin{array}{l}\text { Cholesterol/HDL ratio } \\
\text { (upper tertile }>4.61 \text { ) }\end{array}$} & \multicolumn{2}{|c|}{$\begin{array}{l}\text { Fibrinogen (upper } \\
\text { tertile>290 mg/dl) }\end{array}$} \\
\hline & $\begin{array}{l}\text { Yes } \\
(\mathrm{n}=115)\end{array}$ & $\begin{array}{l}\text { No } \\
(n=1798)\end{array}$ & $\begin{array}{l}\text { Yes } \\
(\mathrm{n}=851)\end{array}$ & $\begin{array}{l}\text { No } \\
(n=1062)\end{array}$ & $\begin{array}{l}\text { Yes } \\
(\mathrm{n}=531)\end{array}$ & $\begin{array}{l}\text { No } \\
(\mathrm{n}=1382)\end{array}$ & $\begin{array}{l}\text { Yes } \\
(\mathrm{n}=664)\end{array}$ & $\begin{array}{l}\text { No } \\
(\mathrm{n}=1249)\end{array}$ & $\begin{array}{l}\text { Yes } \\
(\mathrm{n}=649)\end{array}$ & $\begin{array}{l}\text { No } \\
(n=1264)\end{array}$ \\
\hline effort-reward ratio $(>1)(n=655)$ & $\begin{array}{l}45.2 \\
\chi^{2}=6.55^{\star \star}\end{array}$ & 33.5 & $\begin{array}{l}36.7 \\
\chi^{2}=4.00^{\star}\end{array}$ & 32.3 & $\begin{array}{l}37.1 \\
\chi^{2}=2.67\end{array}$ & 33.1 & $\begin{array}{l}38.7 \\
\chi^{2}=8.65^{\star \star}\end{array}$ & 32.0 & $\begin{array}{l}36.5 \\
\chi^{2}=2.26\end{array}$ & 33.1 \\
\hline Intrinsic effort: & & & & & & & & & & \\
\hline Immersion (upper tertile) $(n=607)$ & $\begin{array}{l}29.6 \\
\chi^{2}=0.27\end{array}$ & 31.9 & $\begin{array}{l}31.8 \\
\chi^{2}=0.01\end{array}$ & 31.6 & $\begin{array}{l}32.6 \\
\chi^{2}=0.24\end{array}$ & 31.4 & $\begin{array}{l}34.6 \\
\chi^{2}=4.19^{\star}\end{array}$ & 30.1 & $\begin{array}{l}31.0 \\
\chi^{2}=0.26\end{array}$ & 32.1 \\
\hline high extrinsic effort (above median) & $\begin{array}{l}47.0 \\
\chi^{2}=0.00\end{array}$ & 47.1 & $\begin{array}{l}47.7 \\
\chi^{2}=0.23\end{array}$ & 46.6 & $\begin{array}{l}49.3 \\
\chi^{2}=1.48\end{array}$ & 46.2 & $\begin{array}{l}47.4 \\
\chi^{2}=0.04\end{array}$ & 46.9 & $\begin{array}{l}48.8 \\
\chi^{2}=1.20\end{array}$ & 46.2 \\
\hline low reward (above median) & $\begin{array}{l}54.8 \\
\chi^{2}=5.04^{\star}\end{array}$ & 44.0 & $\begin{array}{l}45.7 \\
\chi^{2}=0.64\end{array}$ & 43.9 & $\begin{array}{l}46.0 \\
\chi^{2}=0.47\end{array}$ & 44.2 & $\begin{array}{l}49.8 \\
\chi^{2}=11.12^{\star \star}\end{array}$ & 41.9 & $\begin{array}{l}47.6 \\
\chi^{2}=3.38\end{array}$ & 43.2 \\
\hline Women & \multicolumn{10}{|c|}{ Cardiovascular risk factors } \\
\hline \multirow[t]{2}{*}{ Psychosocial variables } & \multicolumn{2}{|c|}{$\begin{array}{l}\text { Hypertension }(\geqslant 160 / 95 \\
m m \mathrm{Hg})\end{array}$} & \multicolumn{2}{|c|}{$\begin{array}{l}\text { Total cholesterol (upper } \\
\text { tertile } \geqslant 220 \mathrm{mg} / \mathrm{dl} \text { ) }\end{array}$} & \multicolumn{2}{|c|}{$\begin{array}{l}\text { LDL-cholesterol (upper } \\
\text { tertile } \geqslant 130 \mathrm{mg} / \mathrm{dl} \text { ) }\end{array}$} & \multicolumn{2}{|c|}{$\begin{array}{l}\text { Cholesterol/HDL ratio } \\
\text { (upper tertile }>3.4 \text { ) }\end{array}$} & \multicolumn{2}{|c|}{$\begin{array}{l}\text { Fibrinogen (upper } \\
\text { tertile>300 } \mathrm{mg} / \mathrm{dl} \text { ) }\end{array}$} \\
\hline & $\begin{array}{l}\text { Yes } \\
(n=69)\end{array}$ & $\begin{array}{l}\text { No } \\
(n=1445)\end{array}$ & $\begin{array}{l}\text { Yes } \\
(n=483)\end{array}$ & $\begin{array}{l}\text { No } \\
(n=1031)\end{array}$ & $\begin{array}{l}\text { Yes } \\
(n=529)\end{array}$ & $\begin{array}{l}\text { No } \\
(n=985)\end{array}$ & $\begin{array}{l}\text { Yes } \\
(n=511)\end{array}$ & $\begin{array}{l}\text { No } \\
(n=1042)\end{array}$ & $\begin{array}{l}\text { Yes } \\
(n=533)\end{array}$ & $\begin{array}{l}\text { No } \\
(n=981)\end{array}$ \\
\hline effort-reward ratio $(>1)(n=438)$ & $\begin{array}{l}39.1 \\
\chi^{2}=3.66\end{array}$ & 28.4 & $\begin{array}{l}30.2 \\
\chi^{2}=0.58\end{array}$ & 28.3 & $\begin{array}{l}28.7 \\
\chi^{2}=0.01\end{array}$ & 29.0 & $\begin{array}{l}27.2 \\
\chi^{2}=1.25\end{array}$ & 30.0 & $\begin{array}{l}29.3 \\
\chi^{2}=0.05\end{array}$ & 28.7 \\
\hline Intrinsic effort: & & & & & & & & & & \\
\hline Immersion (upper tertile) $(\mathrm{n}=512)$ & $\begin{array}{l}31.9 \\
\chi^{2}=0.12\end{array}$ & 33.9 & $\begin{array}{l}36.2 \\
\chi^{2}=1.85\end{array}$ & 32.7 & $\begin{array}{l}37.1 \\
\chi^{2}=3.80^{\star}\end{array}$ & 32.1 & $\begin{array}{l}33.9 \\
\chi^{2}=0.00\end{array}$ & 33.9 & $\begin{array}{l}34.1 \\
\chi^{2}=0.04\end{array}$ & 33.6 \\
\hline high extrinsic effort (above median) & $\begin{array}{l}46.6 \\
\chi^{2}=2.06\end{array}$ & 37.8 & $\begin{array}{l}39.3 \\
\chi^{2}=0.41\end{array}$ & 37.6 & $\begin{array}{l}37.6 \\
\chi^{2}=0.11\end{array}$ & 38.5 & $\begin{array}{l}35.4 \\
\chi^{2}=2.59\end{array}$ & 39.7 & $\begin{array}{l}36.8 \\
\chi^{2}=0.69\end{array}$ & 38.9 \\
\hline low reward (above median) & $\begin{array}{l}60.9 \\
\chi^{2}=9.35^{\star \star}\end{array}$ & 42.2 & $\begin{array}{l}48.2 \\
\chi^{2}=7.75^{\star \star}\end{array}$ & 40.6 & $\begin{array}{l}46.9 \\
\chi^{2}=4.83^{\star}\end{array}$ & 41.0 & $\begin{array}{l}43.6 \\
\chi^{2}=0.13\end{array}$ & 42.7 & $\begin{array}{l}44.7 \\
\chi^{2}=0.85\end{array}$ & 42.2 \\
\hline
\end{tabular}

${ }^{\star} \mathrm{p} \leqslant 0.05,{ }^{\star \star} \mathrm{p} \leqslant 0.01,{ }^{\star \star \star} \mathrm{p} \leqslant 0.001$.

Table 4 Logistic regression analyses: effort-reward ratio and immersion by cardiovascular risk factors among a sample of employed Swedish men and women aged 30-55 years (multivariate odds ratios (95\% CI))

\begin{tabular}{|c|c|c|c|c|c|}
\hline Models* & $\begin{array}{l}\text { Hypertension }(\geqslant 160 / 95 \\
m m \mathrm{Hg})(n=115)\end{array}$ & $\begin{array}{l}\text { Total cholesterol } \\
(\geqslant 220 \mathrm{mg} / \mathrm{dl}) \\
(n=851)\end{array}$ & $\begin{array}{l}\text { LDL-cholesterol } \\
(\geqslant 160 \mathrm{mg} / \mathrm{dl}) \\
(n=531)\end{array}$ & $\begin{array}{l}\text { Cholesterol/HDL ratio } \\
\text { (upper tertile }>4.61) \\
(n=664)\end{array}$ & $\begin{array}{l}\text { Fibrinogen (upper } \\
\text { tertile }>290 \\
m g / d l)(n=649)\end{array}$ \\
\hline \multicolumn{6}{|l|}{ Model I: } \\
\hline effort-reward ratio $(>1)(n=655)$ & $1.69(1.13,2.53)$ & $1.23(1.00,1.50)$ & $1.18(0.95,1.47)$ & $1.31(1.06,1.61)$ & $1.19(0.96,1.47)$ \\
\hline Immersion (upper tertile) $(\mathrm{n}=607)$ & $0.84(0.55,1.31)$ & $0.98(0.80,1.21)$ & $1.04(0.83,1.30)$ & $1.16(0.94,1.44)$ & $0.90(0.72,1.13)$ \\
\hline \multicolumn{6}{|l|}{ Model II: } \\
\hline effort-reward ratio $(>1)(n=655)$ & $1.62(1.07,2.43)$ & $1.20(0.98,1.47)$ & $1.13(0.91,1.41)$ & $1.26(1.02,1.56)$ & $1.13(0.91,1.41)$ \\
\hline Immersion (upper tertile) $(\mathrm{n}=607)$ & $0.83(0.53,1.29)$ & $0.97(0.79,1.19)$ & $0.98(0.78,1.24)$ & $1.12(0.90,1.39)$ & $0.88(0.71,1.11)$ \\
\hline \multicolumn{6}{|l|}{ Women $(n=1514)$} \\
\hline Models* & $\begin{array}{l}\text { Hypertension }(\geqslant 160 / 95 \\
m m \mathrm{Hg})(n=69)\end{array}$ & $\begin{array}{l}\text { Total cholesterol } \\
(\geqslant 220 \mathrm{mg} / \mathrm{dl}) \\
(n=483)\end{array}$ & $\begin{array}{l}\text { LDL-cholesterol } \\
(\geqslant 130 \mathrm{mg} / \mathrm{dl}) \\
(n=529)\end{array}$ & $\begin{array}{l}\text { Cholesterol/HDL ratio } \\
\text { (upper tertile }>4.61) \\
(n=511)\end{array}$ & $\begin{array}{l}\text { Fibrinogen (upper } \\
\text { tertile }>300 \\
m g / d l)(n=533)\end{array}$ \\
\hline \multicolumn{6}{|l|}{ Model I: } \\
\hline effort-reward ratio $(>1)(n=438)$ & $1.57(0.92,2.66)$ & $0.99(0.76,1.28)$ & $0.87(0.68,1.13)$ & $0.84(0.65,1.09)$ & $1.02(0.78,1.31)$ \\
\hline Immersion (upper tertile) $(\mathrm{n}=512)$ & $0.84(0.48,1.45)$ & $1.26(0.98,1.61)$ & $1.37(1.07,1.74)$ & $1.04(0.81,1.33)$ & $1.02(0.80,1.30)$ \\
\hline \multicolumn{6}{|l|}{ Model II: } \\
\hline effort-reward ratio $(>1)(n=438)$ & $1.56(0.92,2.66)$ & $0.98(0.76,1.28)$ & $0.88(0.68,1.13)$ & $0.86(0.66,1.11)$ & $1.03(0.79,1.33)$ \\
\hline Immersion (upper tertile) $(n=512)$ & $0.84(0.48,1.45)$ & $1.26(0.98,1.62)$ & $1.39(1.09,1.77)$ & $1.08(0.84,1.39)$ & $1.03(0.81,1.32)$ \\
\hline
\end{tabular}

^Model I: adjusted for age and behavioural risk factors (regular cigarette smoking $>10 \mathrm{cig} /$ day, BMI $\geqslant 27$, lack of physical exercise $<2 /$ week). Model II: adjusted for age, behavioural risk factors, biomedical risk factors (depending on the outcome hypertension or total cholesterol; fibrinogen: hypertension) and socioeconomic group (white $v$ blue collar).

accordance with a system used in Swedish censuses. For the purpose of this report subjects were categorised as blue collar workers or white collar workers.

STATISTICAL METHODS

Univariate and bivariate methods were used to characterise the study population in terms of frequency or mean value and standard deviation of psychosocial and biobehavioural variables and cardiovascular risk factors. Statistical significance of bivariate asociations was calculated with the help of $\chi^{2}$ or two sided $t$ tests. Multivariate logistic regression analysis was used to control for confounders in examining the relation of indicators of effort-reward imbalance and cardiovascular risk factors. Logistic regression analysis as the most appropriate statistical method was chosen because of the deviation from normal distribution of the effort-reward ratio. ${ }^{24}$ The likelihood ratio difference test was used to check the fit of each model accepting a significance of $10 \%$ to avoid second order errors. Multivariate prevalence odds ratios (POR) and 95\% confidence intervals derived from the logistic regression models are given in respective tables. 
Model I $(F=3.67, \mathrm{p}=0.026)$

Model II $(F=3.76, \mathrm{p}=0.024)$

Model I adjusted for age.

Model II most parsimonious model adjusted for age, BMI, cigarette smoking.

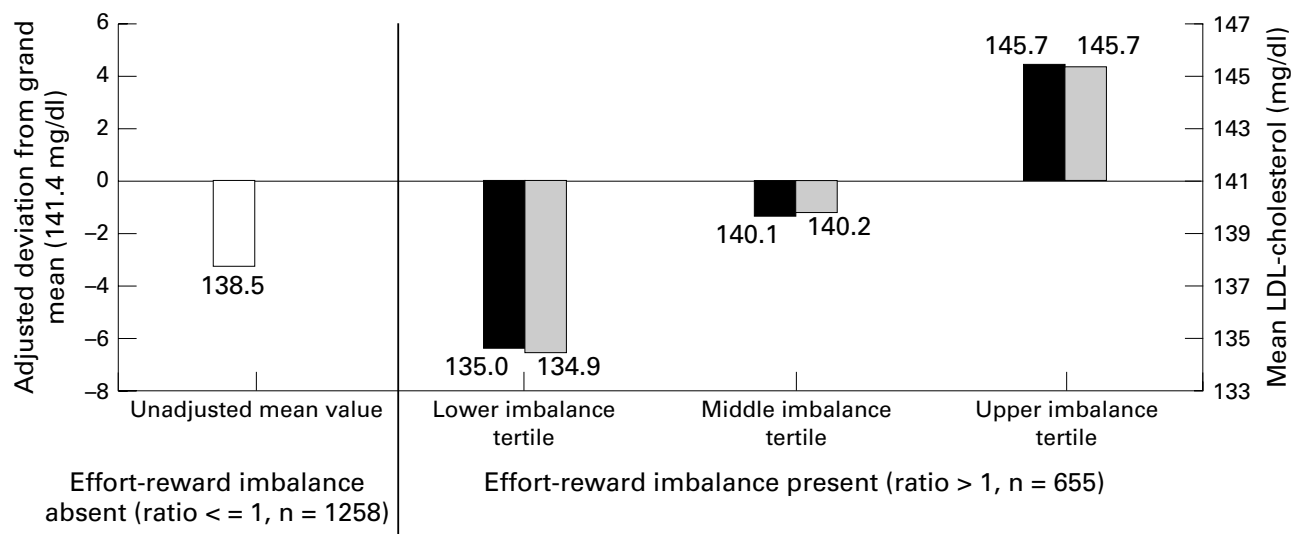

Figure 1 Intensity of effort-reward imbalance and mean LDL-cholesterol values in a sample of 655 Swedish men aged 30-55 years.

Model I: $F=4.50, \mathrm{p}=0.034$

Model II: $F=4.73, \mathrm{p}=0.03$

Model I adjusted for age.

Model II most parsimonious model adjusted for age, BMI, cigarette smoking.

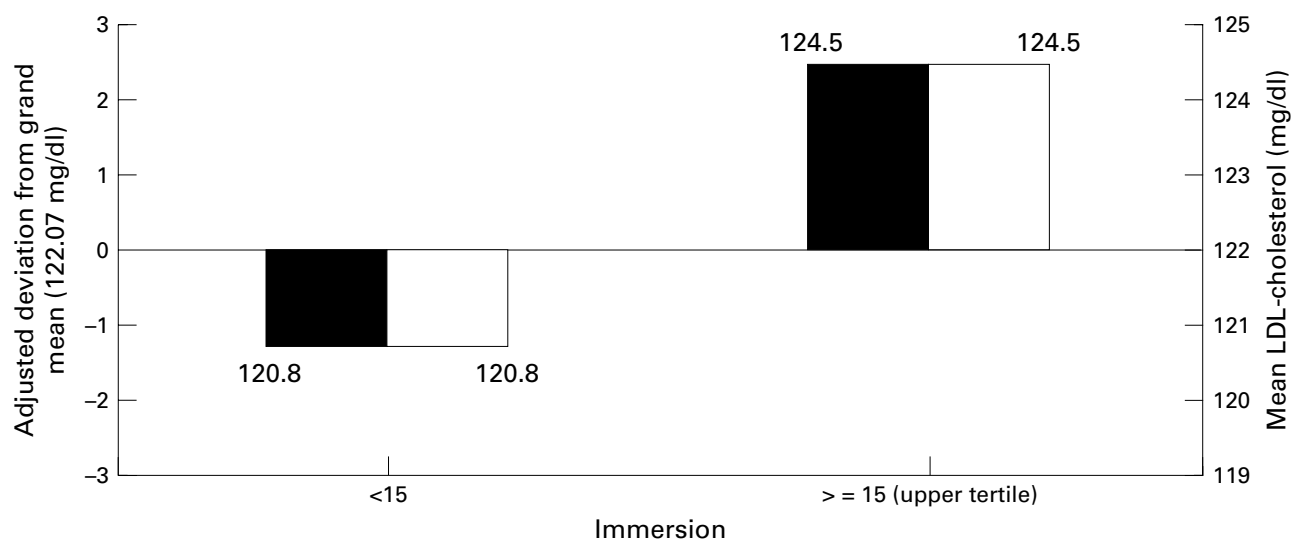

Figure 2 Intrinsic effort and mean LDL-cholesterol values in a sample of 1514 Swedish women aged 30-55 years.

Analysis of variance (ANOVA) was performed to test the relation between indicators of high effort and low reward and mean values of LDL-cholesterol. Among men the relation of the effort-reward ratio and mean LDL- cholesterol values was analysed. Only men with an imbalance between extrinsic effort and reward (effort-reward ratio $>1$ ) were included in this analysis to test the association between the intensity of effort-reward imbalance and the cardiovascular risk factor. Among women the relation between high levels of intrinsic effort (upper tertile of immersion) and mean LDLcholesterol was tested. The choice of analysis of variance was justified for two reasons. Firstly, the use of tertiles was the most appropriate way to estimate the intensity of effort-reward imbalance in terms of ratio values $>1$. The distribution of the data did not allow to calculate statistical analysis with continuous ratio values because of deviation from normal distri- bution. Secondly, LDL-cholesterol in all categories of the factor variables does not significantly deviate from normal distribution as tested by the Kolmogorov-Smirnov test.

All statistical analyses were carried out using SPSS/PC for Windows. ${ }^{25}$ All analyses were perfomed for men and women separately. Two sided tests of significance with values of $p<$ 0.05 were considered statistically significant.

\section{Results}

Table 1 describes the study sample in terms of biobehavioural and psychosocial characteristics. Men and women differ significantly with regard to all biobehavioural and psychosocial factors listed in the table with the exception of age. Women have lower mean values of blood pressure, total cholesterol, LDL-cholesterol, and the effort-reward ratio compared with men whereas fibrinogen and HDL-cholesterol and 
immersion are higher in women. With regard to socioeconomic group an increased proportion of women belong to the white collar stratum.

In table 2 the prevalence of indicators of effort-reward imbalance is presented according to selected sociodemographic characteristics. There is a clear trend towards a higher prevalence of a mismatch between extrinsic effort and reward (ratio $>1$ ) and an increased level of immersion among men with higher education and training and of those belonging to the white collar part of the study sample. In women results indicate critical effort-reward ratio values in those in the higher age groups, those with higher education, and in the white collar workers. Immersion is also more prevalent in female white collar workers.

Table 3 shows the prevalence of high scores of extrinsic effort, low reward, of the effortreward ratio and of high intrinsic effort (immersion) in men and women with and without cardiovascular risk factors. Different patterns of associations between risk factors and psychosocial variables can be observed with regard to sex. Whereas high scores on the effort-reward ratio are more prevalent among men with cardiovascular risk factors (especially hypertension, total cholesterol, cholesterol/ HDL ratio) immersion is more prevalent in women with increased total cholesterol and an increased cholesterol/HDL ratio. Moreover, the mismatch between high effort and low reward (effort reward ratio $>1$ ) discriminates better between men with and without cardiovascular risk factors than the presence of either high effort or low reward.

To test the main hypothesis of a relation between of indicators of effort-reward imbalance and cardiovascular risk factors multivariate logistic regression analyses were performed. As can be seen from table 4, critical scores of the effort-reward ratio are related to hypertension, total cholesterol, and the cholesterol/ HDL ratio in men (POR ranging from 1.23 to 1.69) even after adjustment for relevant confounders. No comparable effects can be observed with regard to immersion among men. In women immersion is independently associated with LDL-cholesterol (POR 1.37, 1.39) and tends to be related to increased total cholesterol (POR 1.26). Although the prevalence odds ratios of effort-reward imbalance with regard to hypertension are the highest for women they are not within a statistically acceptable range. This is because of the small numbers of women with hypertension (see table 3). In summary, among men effortreward imbalance measured by the ratio between extrinsic effort and reward is associated with cardiovascular risk factors whereas in women a relation between immersion and LDL-cholesterol is observed. These effects hold true after adjustment for relevant confounders.

Finally, as indicated in the Methods section, analyses of variance (ANOVA) were performed to test associations between indicators of high effort and low reward and LDL-cholesterol using the cardiovascular risk factor as a continuous outcome variable.

Regarding men the relation between the effort-reward ratio and LDL-cholestrol was tested. The effort-reward ratio was subdivided into tertiles indicating that subjects in the upper tertile suffer from the most intensive effortreward imbalance. Figure 1 shows, that LDLcholesterol increases with an increasing intensity of effort-reward imbalance (model I: $F=3.69$, $\mathrm{p}=0.026$, model II: $F=3.76, \mathrm{p}=0.024)$ even after adjustment for relevant confounders. In women the association between immersion and LDLcholesterol was tested. As can be seen from figure 2 the mean value of LDL-cholesterol is increased for those women who have high levels of intrinsic effort (upper tertile of immersion). This association holds true after adjustment for confounders (Model I: $F=4.5, \mathrm{p}=0.034$; Model II: $F=4.73$, p=0.030).

\section{Discussion}

The relation between chronic work stress in terms of effort-reward imbalance and cardiovascular risk factors (that is, hypertension, increased atherogenic lipids, increased fibrinogen) was examined in a study on 3427 healthy employed men and women aged 30-55 years living in the greater Stockholm area. After adjustment for relevant confounders results of multivariate logistic regression analyses showed sex differences in the association between two indicators of effort-reward imbalance and cardiovascular risk factors. Results indicated in men an increased risk of being hypertensive and of having increased total cholesterol as well as an unfavourable total cholesterol/HDL ratio for those reporting higher extrinsic efforts than rewards. In women a measure of intrinsic effort at workimmersion - was independently related to increased LDL-cholesterol level. Analyses of variance (ANOVA) confirmed an association between the effort-reward ratio and level of LDL-cholesterol in men (see fig 1) and an association of immersion with LDLcholesterol in women (see fig 2).

Additional support in favour of the reported sex differences can be derived from results not presented in detail. They suggest that men with increased cardiovascular risk factors are more likely to suffer from high extrinsic effort (in particular time pressure) and low status control (for example, job insecurity and undesirable changes in the work situation), whereas women with increased cardiovascular risk factors were more likely to experience insufficient esteem by superiors and lack of reciprocal support. These results too can be interpreted in the framework of gender role-specific expectations regarding work and career, as indicated above.

Nevertheless, the following limitations of the reported findings need to be taken into account. Firstly, the results are restricted because of the cross sectional design of the study, which precludes the testing of causal relations.

Secondly, in this study the assessment of effort-reward imbalance was not completely identical with the original measures, mainly 
because of economic constraints in view of a large sample size. The few numbers of items measuring extrinsic effort and reward may be responsible for the relatively low internal consistency of respective scales (see appendix 1). With few numbers of items in a scale only relatively low Cronbach's $\alpha$ values can be expected, especially if these items are dichotomous as was the case with the items measuring extrinsic effort and reward. ${ }^{26}$ These limitations may be responsible for the relatively low prevalence odds ratios in this study compared with previous findings. ${ }^{27}{ }^{28}$ Nevertheless, proxy indicators of effort-reward imbalance turned out to be valid measures that predict increased cardiovascular risk as well as high symptom scores independent from personal traits like negative affectivity. ${ }^{29} 30$ Moreover, other measures of perceived work stress like low job control are also related to cardiovascular risk even after adjustment for personal traits. In a prospective study the association between low job control and risk of coronary heart disease was not explained by negative affectivity. ${ }^{31}$

Thirdly, the restriction of the analyses to people with complete data may have caused an underestimation of the reported relations. People excluded from analyses because of unsystematic missing data had a significantly higher prevalence of hypertension, increased total cholesterol, unfavourable levels of the total cholesterol/HDL-cholesterol ratio, and a higher prevalence of effort-reward ratio values $>1$.

One further limitation concerns the influ- ence of additional confounders not included inthe present analysis such as alcohol consumption and diet. In a previous study we found that neither variable invalidated the observed association of effort-reward imbalance with cardiovascular risk. ${ }^{27} 28$

Finally, the validity of our measures of chronic work stress may be limited because of the fact that subjects suffering from cardiovascular risk may overestimate reported work stress. This is most probable in the case of known history of hypertension. ${ }^{32}$ To rule out this bias we repeated multivariate analyses of the association between effort-reward imbalance and hypertension adjusting for the information whether subjects were aware or unaware respectively of their hypertensive status. The magnitude of the association decreased a little but was still significant.

Despite the discussed limitations we maintain that the findings add to the accumulated evidence from the literature that work stress in terms of effort-reward imbalance is related to cardiovascular risk in middle aged working populations. Moreover, results showed gender differences in associations of effort-reward imbalance with cardiovascular risk factors. In view of the public health implications of these findings, further research efforts including intervention approaches are desirable.

Funding: the analysis of data presented in the manuscript and the scientific exchange crucial to the findings were part of the BIOMED 1 "Heart at Work Network", which was funded by the European Union 1994 to 1997 (CT94-1065).

Conflicts of interest: none.

Appendix 1 Measures of extrinsic and intrinsic effort and reward in the WOLF-study (factor loadings in parentheses, Cronbach's alpha)

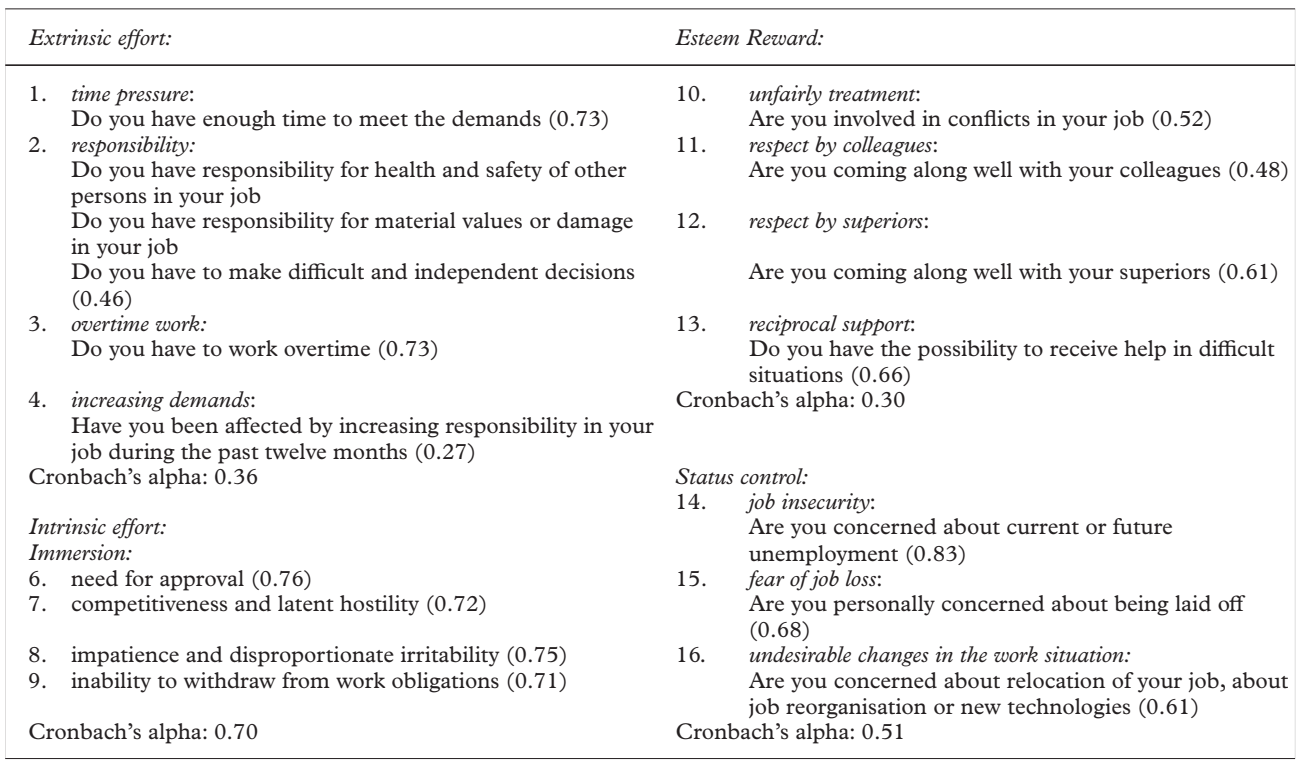

Items measuring extrinsic effort or reward, and the four subscales measuring intrinsic effort, were added to three factor based scales according to our theory. Main component factor analysis was performed to examine the dimensions of the effort and reward items. Cronbach's alpha was calculated to estimate the internal consistency of the factor based scales. The results of the factor and reliability analyses are not shown in detail in this paper.

The construction of the effort-reward ratio as described in the Methods section was restricted to those indicators of extrinsic effort and reward that exhibit a prevalence of at least $20 \%$ of distressed subjects, reporting that they are often or very often concerned by the stressor. This criterion was fulfilled by three measures of extrinsic effort (that is, time pressure, overtime work, responsibility) and four measures of low reward (that is, respect by colleagues, reciprocal support, undesirable change in the work situation, job insecurity). This procedure was chosen to concentrate the analyses to highly prevalent stressful experience at work. 
1 French JR, Caplan RD, Harrison RV. The mechanisms of job stress and strain. Chichester, England: Wiley, 1982.

2 House JS. Work stress and social support. Reading, MA. Addison Wesley, 1981.

3 Theorell T, Karasek R. Current methodological issues relating to psychosocial job strain and cardiovascular disease research. F Occup Health Psychol 1996;1:9-26.

$4 \mathrm{Kasl}$ S. Influence of the work environment on cardiovascular health: a historical, conceptual, and methodological perspective. F Occup Health Psychol 1996;1:42-56.

5 Siegrist J. Adverse health effects of high-effort/low-reward conditions. F Occup Health Psychol 1996;1:27-41.

6 Karasek RA. Job demands, job decision latitude, and mental strain: implications for job redesign. Administration Science Quaterly 1979;24:285-307.

7 Karasek RA, Theorell T. Healthy work: stress, productivity, and the reconstruction of working life. New York: Basic Books, 1990.

8 Johnson JV, Hall E. Job strain, work place social support, and cardiovascular disease. Am ₹ Public Health 1988;78:133642 .

9 Johnson JV, Hall E, Theorell T. Combined effects of job strain and social isolation on cardiovascular disease morbidity and mortality in a random sample of the Swedish male working population. Scand $\mathcal{F}$ Work Environ Health 1989;15:271-9.

10 Schnall PL, Landsbergis PA, Baker D. Job strain and cardiovascular disease. Annu Rev Public Health 1994;15 381-411.

11 Hall EM. Gender, work control and stress: A theoretical discussion and an empirical test. Int f Health Serv 1989;19: 725-45.

12 Hall, EM. Double exposure: The combined impact of the home and work environments on mental strain and physical illness. Int 7 Health Serv 1992;22:239-60.

13 Eaker ED, Prisky J, Castelli WP. Myocardial infarction and coronary death among women: psychosocial predictors from a 20 -year follow-up in the Framingham Study. Am f Epidemiol 1992;135:854-64.

14 Henry JP, Stephens PM. Stress, health, and the social environment. Berlin: Springer, 1977.

15 Beamish RE, Singal PK, Dhalla NS, eds. Stress and heart disease. Boston: Martinus Nijhoff, 1985.

16 Matschinger H, Siegrist J, Siegrist K, et al. Type A as a coping career: towards a conceptual and methodological redefinition. In: Schmidt TH, Dembroski TM, Blümchen $\mathrm{G}$, eds. Biological and psychological factors in cardiovascula disease. Berlin: Springer, 1986:104-26.

17 Siegrist J, Peter R, Junge A, et al. Low status control, high effort at work and ischemic heart disease: prospective evidence from blue-collar men. Soc Sci Med 1990;31:112734.
18 Expert Panel on Detection, Evaluation, and Treatment of High Blood Cholesterol in Adults. Summary Of the Second Report of the National Cholesterol Education Program (NCEP) Expert Panel on Detection, Evaluation, and Treatment of High Blood Cholesterol in Adults (Adult Treatment Panel II). FAMA 1993;269:3015-23.

19 Castelli WP, Anderson KA. A population at risk. Am f Med 1986;80 (suppl 2A):23-32.

20 Baker IA, Eastham R, Elwood PC, et al. Haemostatic factors associated with ischemic heart disease in men aged 45-64 years: the Speedwell Study. Br Heart f 1982;47:490-4

21 Wilhelmsen L, Svärdsudd K, Korsan-Bengtsen K, et al. Fibrinogen as a risk factor for stroke and myocardial infarction. N Engl f Med 1984;311:501-5.

22 Folsom AR, Wu KK, Davies CE, et al. Population correlates of plasma fibrinogen and factor VII: putative cardiovascular risk factors. Atherosclerosis 1991;91:191-205.

23 Brunner E, Smith GD, Marmot M, et al. Childhood social circumstances and psychosocial and behavioural factors as determinants of plasma fibrinogen. Lancet 1996;347:100813.

24 Efron B. The efficiency of logistic regression compared to normal discriminant analysis. F Am Statist Assoc 1975;70: 892-8.

25 Norusis MJ. SPSS for Windows Release 6.0. Chicago: SPSS Inc, 1993

26 Kim JO, Muller CW. Factor analysis -statistical methods and practical issues. Beverly Hills: Sage Publications, 1978.

27 Peter R, Siegrist J. Chronic work stress, sickness absence, and hypertension in middle managers - general or specific sociological explanations? Soc Sci Med 1997;45:1111-20.

28 Siegrist J, Peter R, Cremer P, et al. Chronic work stress is associated with atherogenic lipids and elevated fibrinogen in middle-aged men. F Intern Med 1997;242:149-56.

29 Bosma H, Peter R, Siegrist J, et al. Alternative Job stress models and the risk of coronary disease: the effort-reward imbalance model and the job strain model. Am f Public Health 1998;88:68-74.

30 Peter R, Geißler H, Siegrist J. Associations of effort-reward imbalance at work and reported symptoms in different groups of male and female public transport workers. Stress Medicine (in press).

31 Bosma H, Marmot MG, Hemmingway H, et al. Low job control and risk of coronary heart disease in Whitehall II (prospective cohort) study. BMF 1997;314:558-65.

32 Theorell T. Family history of hypertension —an individual trait interacting with spontaneously occuring job stressors. Scand F Work Environ Health 1990;16 (suppl 1):74-9. 